\title{
EVALUACIÓN DEL APRENDIZAJE: LA RAZÓN PARA LA FORMACIÓN INTEGRAL DEL POLICÍA
}

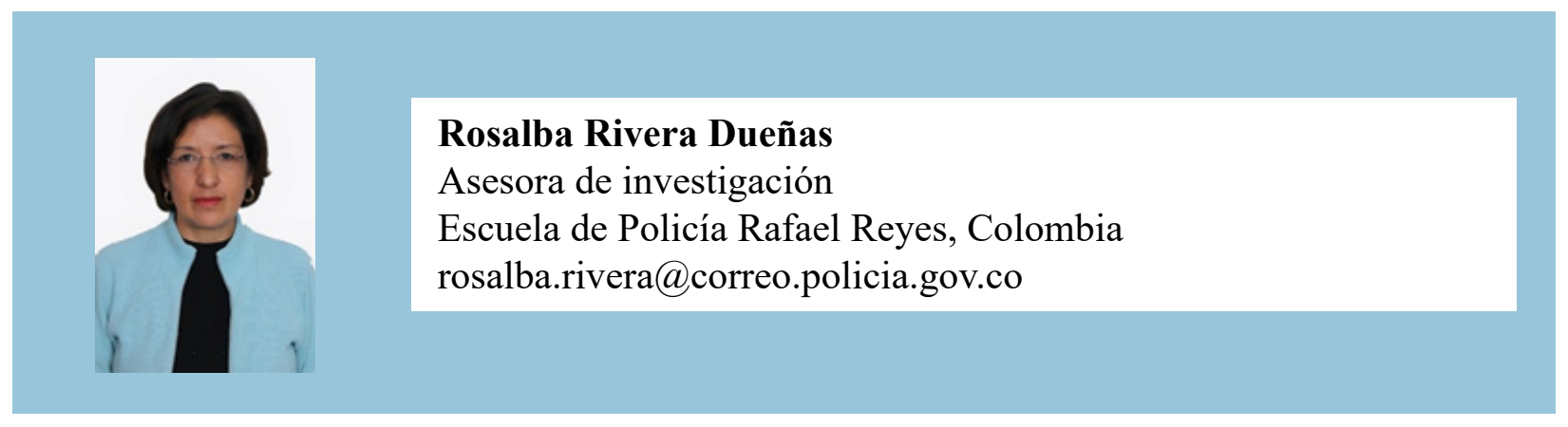

\section{RESUMEN}

El escrito tiene como finalidad narrar el procedimiento de evaluación del aprendizaje en una escuela de formación de la Policía Nacional de Colombia, teniendo en cuenta que la formación integral en la educación de los seres humanos y su validación se basa en un principio denominado "evaluación del aprendizaje”, del cual nació el interés de conocer su procedimiento y es así como a través de una investigación exhaustiva se determinaron las concepciones y prácticas evaluativas predominantes en la acción pedagógica de los docentes de la escuela de Policía Rafael Reyes, propendiendo por la actualización y mejoramiento de la práctica evaluativa acorde a los nuevos desafíos del sistema educativo. El artículo contiene información relevante de carácter cuantitativo y cualitativo de sondeo respectivamente, que se utilizó durante la investigación, para que la información recolectada se complemente y se contraste, debido a la diversidad de la interpretación de los resultados. Finalmente, se presenta un plan de mejoramiento institucional que conllevó al desarrollo de estrategias de capacitación y actualización, lo cual ha contribuido a la innovación de los procesos de evaluación del aprendizaje de los estudiantes en la institución educativa de la policía y el propósito es que sea aplicado a todas las unidades educativas de la Policía Nacional.

Palabras clave: Educación, educación integral, principios, evaluación del aprendizaje y estrategias. 


\title{
EVALUATION OF LEARNING: THE REASON FOR THE COMPREHENSIVE TRAINING OF THE POLICE
}

\begin{abstract}
The brief aims to narrate the evaluation process of learning a training school of the National Police of Colombia, considering that the comprehensive training in the education of human beings and their validation is based on a principle called "assessment learning ", which was born the interest to know their procedure and is as well as through extensive research concepts and prevailing assessment practices in the pedagogical action of teachers school Police Rafael Reyes, inclining by the update were determined and improvement of evaluation according to the new challenges of the education system practice. The article contains information relevant quantitative and qualitative probing respectively, which was used during the investigation, so that the collected information is supplemented and contrast, due to the diversity of the interpretation of the results.Finally, an institutional improvement plan that led to the development of training strategies and updating is presented, which has contributed to the process innovation learning assessment of students in the school police and the purpose is to be applied to all educational units of the National Police.
\end{abstract}

Keywords: education, comprehensive education, principles, assessment of learning, Strategies.

\section{INTRODUCCIÓN}

La educación como la capacidad para desarrollar la inteligencia y la posibilidad de aprender y crear saberes aplicables a la vida; teniendo en cuenta esta premisa, un grupo de investigadores de una escuela de formación de la Policía Nacional, adelantó un proyecto de investigación, abordando el tema sobre concepciones y prácticas evaluativas que predominan en la acción pedagógica de los docentes, el cual tuvo como objetivo, identificar las prácticas evaluativas que predominan en las acción educativas de los docentes, con el propósito de establecer un plan de mejoramiento institucional que conllevará al desarrollo de estrategias de capacitación y actualización que contribuyan a la innovación de los procesos de evaluación del aprendizaje de los estudiantes.

En este escrito, realizaré un recuento, de lo que fue el proceso y los resultados de la investigación, adelantada por Rivera (2015a) a través de tres (3) etapas: En la primera, se 
identificaron los aciertos y desaciertos de los conceptos y habilidades evaluativas que los docentes de la escuela utilizan en su ejercicio pedagógico, en la segunda se categorizaron los conceptos y prácticas que más utilizan los docentes frente al proceso de evaluación del aprendizaje y luego se planteó una estrategias de fortalecimiento para la evaluación del aprendizaje de los docentes de la Escuela de Policía Rafael Reyes.

Este estudio permitió diseñar una estrategia didáctica para facilitar la evaluación del aprendizaje por medio de una metodología clara y sencilla, que permita fortalecer la educación integral en los aspirantes a Técnicos Profesionales de la Policía Nacional.

\section{TEORÍAS FUNDAMENTALES}

El trabajo de investigación se fundamentó en teorías sobre: Funciones Básicas en la Formación Policial, determinadas como la docencia, la investigación y proyección social, que se encuentran inmersas en la ley de Educación Superior y son correlacionadas entre sí, por tanto, no se puede prescindir de ninguna de ellas.

En lo relativo a los Enfoques Pedagógicos, en la Policía Nacional desde el 2010 se promueve la teoría del constructivismo, el aprendizaje por interacción social y el aprendizaje significativo; en los cuales el constructivismo privilegia el aprendizaje activo y autónomo como estrategia para la construcción del propio conocimiento.

También se hizo referencia a la Evaluación del Aprendizaje, sobre lo cual la licenciada González (2001) refiere que el significado de esta teoría se aproxima a las definiciones generales que se presentan en los diccionarios y consiste en apreciar, valorar, fijar el valor de una cosa, hecho o fenómeno.

\section{METODOLOGÍA}

El estudio se desarrolló bajo una metodología de tipo descriptivo, porque permite identificar las concepciones y prácticas evaluativas que predominan en la acción pedagógica de los docentes de la Escuela de Policía Rafael Reyes y propende por la actualización y mejoramiento de la práctica evaluativa acorde a los nuevos desafíos del sistema educativo. 
Frente a la recolección de la información utilizaron instrumentos de carácter cuantitativo y cualitativo como la encuesta y el cuestionario de sondeo respectivamente, con la intención de que las informaciones se complementen y se contrasten; porque la diversidad de la interpretación de los resultados, amerita el uso de estas técnicas. Rivera (2015b), en el "Manual Académico para estudiantes de la Dirección Nacional de Escuelas", explica que la evaluación de los estudiantes se define sobre la base de evaluación del aprendizaje que demuestra los avances alcanzados por la comunidad educativa en relación con la construcción, aplicación de conocimientos y el desarrollo de las competencias según el modelo descrito en el Sistema Educativo Policial, expresado en calificaciones numéricas y cualitativas. (2010; p.p.16).

Además, describe las características de esta evaluación educativa en la que dice que debe ser integral; es decir, debe ser un proceso continuo, sistemático, coherente, formativo y concertado entre las partes que actúan (docente-estudiante). También trae a relación el propósito de la misma, indicando que la evaluación debe estar orientada a verificar el logro de competencias por parte del estudiante, identificar restricciones o problemas en el proceso de aprendizaje, facilitar información al docente para revisar y ajustar las actividades del aprendizaje, igual que informar al estudiante sobre los resultados obtenidos, rediseñar las estrategias de aprendizaje si es necesario, retroalimentar al estudiante e indicar alternativas de refuerzo y por último verificar la calidad del proceso educativo y aplicar mejora si se requiere.

\section{RESULTADOS OBTENIDOS EN LA INVESTIGACIÓN}

Se identificó el Modelo Pedagógico que maneja la Escuela, descrito en el Capítulo Marco de Referencia, específicamente en el Marco Teórico que a la letra dice: "El Proyecto Educativo Institucional de la Policía Nacional determina la Teoría del Constructivismo, el aprendizaje por interacción social y el aprendizaje significativo. El constructivismo privilegia el aprendizaje activo y autónomo como estrategia para la construcción del propio conocimiento. El aprendizaje por interacción social plantea que la educación es un proceso eminentemente social, donde prevalece el aprendizaje colaborativo y el aprendizaje significativo; afirma que el estudiante se apropia de los conocimientos, los integra a sus aprendizajes previos y estructura mental, para aplicarlos en su desempeño".(Policía Nacional, PEI, 2010).

La educación desarrolla competencias transversales, las cuales fueron identificadas a partir de las competencias genéricas planteadas en el Modelo de Gestión Humana. Las competencias 
transversales hacen referencia a los conocimientos, habilidades y actitudes, que permiten al profesional de policía actuar de manera constructiva en la sociedad y desarrollar condiciones positivas para el desempeño; dentro de las competencias transversales se encuentra:

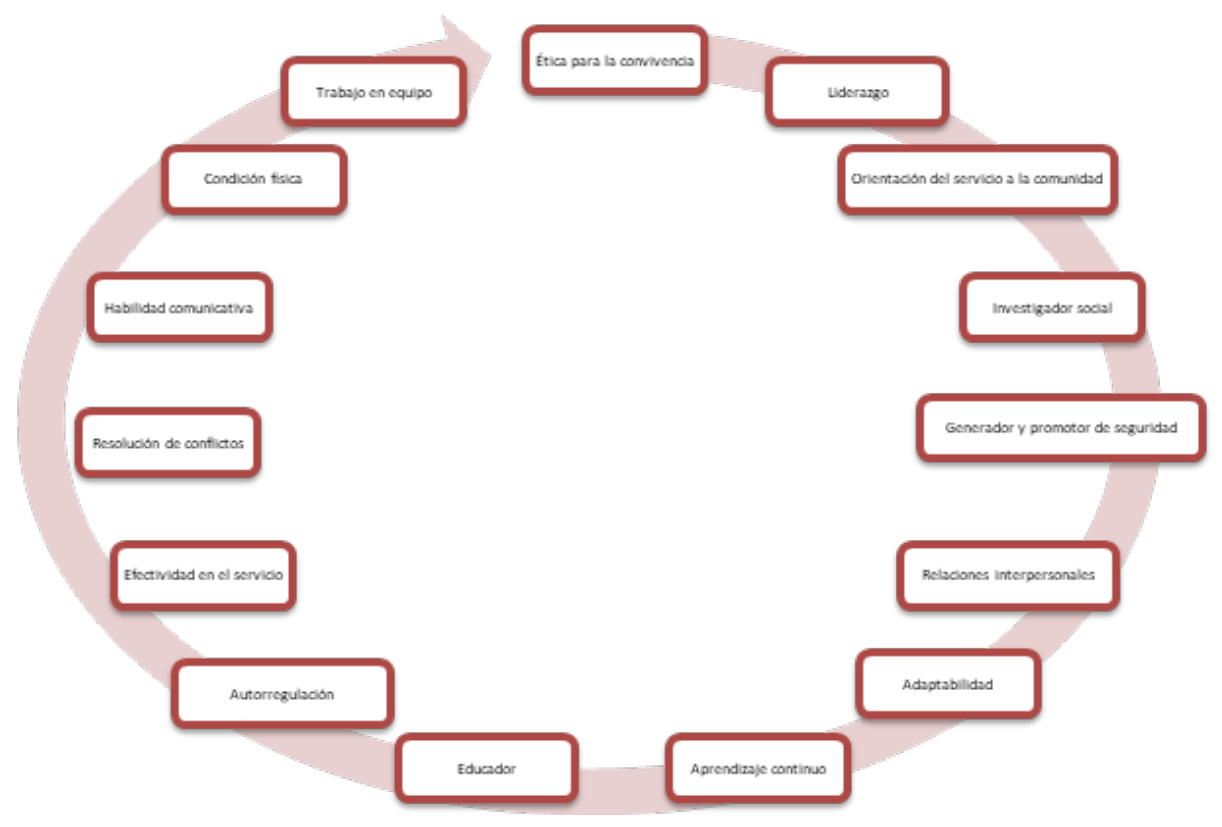

FIGURA 1: Competencias Transversales.

Fuente: Proyecto Educativo Institucional. PEI. Policía Nacional， (2010; p.p. 20).

Claramente el proceso educativo tiene su fuente de organización en la gestión académica, la cual gira en torno al currículo. Esta gestión se lleva a cabo mediante las etapas de planeación, ejecución, evaluación (de programas, estudiantes, docentes, egresados, entre otros) y retroalimentación permanente, en este sentido, y teniendo en cuenta la concepción educativa de la Policía Nacional, el currículo se diseña a partir de varios interrogantes:

¿Qué tipo de persona se pretende formar? Un policía integral y competente. Un ciudadano ejemplar, comprometido con su profesión y con la comunidad.

¿Para qué tipo de sociedad? Para un país con conflicto interno, y en búsqueda de la paz, en un estado social de derecho y democrático.

¿Para qué? Para el logro de unos propósitos educativos, como respuesta a las necesidades institucionales y del país.

¿Cómo? Mediante programas académicos, planes de estudio, procesos, procedimientos y un enfoque pedagógico fundamentado en competencias. 
¿Con qué recursos? Con recursos técnicos, tecnológicos, logísticos y financieros, y su capital más importante: el talento humano.

¿Con quién? Con una comunidad educativa comprometida con la gestión académica y policial.

En donde las características del currículo deben evidenciar la concepción educativa de la institución. Por esto, se plantea una gestión curricular que impregne los espacios educativos, de estrategias de aprendizaje que permitan formar profesionales con los conocimientos, habilidades, destrezas y actitudes, requeridos para desempeñarse de manera exitosa en el servicio policial. En este sentido, el currículo, comprende las siguientes características: es flexible, integral, pertinente, se fundamenta en competencias, además es transversal. Además, la gestión curricular es la columna vertebral del proceso educativo. En este proceso participan directivos, docentes, estudiantes y egresados, para dar cumplimiento a los objetivos propuestos en los diferentes programas académicos. La gestión curricular se cumple mediante las etapas de diseño, desarrollo y evaluación curricular (Policía, 2010).

Es de resaltar que Rivera (2015) indica que para fortalecer el desarrollo de la investigación se deben tomar la tarea de referenciarse en el modelo de evaluación de la Universidad de la región conocida con el nombre de Juan de Castellanos, debido a que el Proyecto Educativo Institucional (PEI) de la Fundación Juan de Castellanos se enmarca en un modelo pedagógico integral, el cual comprende lo holístico, sinérgico e interdisciplinario que recrea y refunde todos los elementos mencionados. Este paradigma tiene un acento personalista cristiano y antropo-ético. A partir del cual se deriva la proyección socio- humanística, que destaca la dimensión cósmica y ecológica. Se apuesta por una educación que potencie y privilegie la armonía: con sí mismo, con los demás, con la naturaleza y con Dios y se apunta hacia la auténtica formación integral, que fortalece la autonomía y la transformación de la persona.

Con el estudio comparativo los investigadores pudieron determinar fortalezas y debilidades frente al proceso de evaluación que los docentes aplican en la escuela, en el que los registros de observación arrojaron resultados positivos frente al proceso de evaluación como que al iniciar el período o la primera clase los docentes informan al estudiante cómo son las políticas de evaluación y dentro de estas explican que se tiene en cuenta la participación en clase, la asistencia a la misma, la realización de un trabajo y un examen final. 
De otra parte, ellos indagaron dentro de los docentes el contexto en cuanto a qué se piensa de la evaluación de los contenidos impartidos en su clase, con el ánimo de apreciar la postura del educador frente al aprendizaje, con resultados para los docentes una actividad necesaria hacia el emprendimiento de docentes y dicentes. Gráficamente se representa de la siguiente manera:

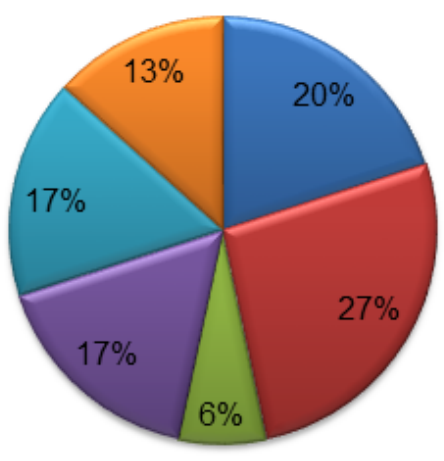

- Hay Interes

- Corregirlos

$\square$ Productividad

- Sistematizar

$\square$ Entendimiento

๑Dinámica de aprendizaje

\section{Gráfica1. Pensamiento de los docentes frente a la evaluación.}

También, conocieron los autores que dentro de las formas de evaluar la mayoría de los docentes practican con exámenes escritos, talleres dentro de las clases y participación en clase, aunque para evaluar el aprendizaje en educación existen otros medios, como los ensayos críticos, análisis de casos, mesas redondas, entre otros.

Lo que es impactante resaltar es que los docentes colocan trabajos en la última semana, es decir sobre el tiempo de terminar el periodo académico, ocasionando traumatismos en los estudiantes, por la acumulación de trabajo, la falta de tiempo para cumplir con todas las tareas, además de las otras labores propias de un policial.

\section{Prácticas de evaluación del aprendizaje}

En cuanto a la segunda etapa, Rivera (2015c) se toman la tarea de priorizar los conceptos y prácticas validas que más utilizan los docentes frente al proceso de evaluación del aprendizaje, encontrando que planifican muy bien las estrategias de evaluación en los planes de trabajo de las asignaturas que orientan, se evidencia en los planes de trabajo, en los que se muestran las estrategias significativas de aprendizaje, Estrategias de Evaluación, Criterios de Evaluación, además están especificadas claramente, en los contenido programáticos de cada asignatura, al 
final de cada tema y del contenido temático de la siguiente manera.

Al concluir cada unidad se especifican las estrategias significativas de aprendizaje con tácticas de debate y análisis de casos, desarrollo de conocimientos fundamentales mediante exposiciones, lectura de textos, relatorías, entre otras, así como de conocimientos procedimentales a través de herramientas didácticas como talleres, representación de casos y exploración de casos con expertos, las habilidades comunicativas mediante debates, foros y construcción de textos para crear discurso propio acerca de la Bioética y ejercicios individuales y grupales que permitan al estudiante construir para sí y para el grupo, en el que el docente debe interactuar con la juventud para orientar su problemática.

Al igual, presenta una matriz para evaluar por competencias, donde el docente debe diligenciarla, de acuerdo a la asignatura y estrategias significativas de aprendizaje, registrando, en la primera columna las competencias citadas en este contenido, en la segunda las estrategias que emplearía el docente para evaluarlas y en la tercera los criterios que considera debe tener en cuenta para evaluar las estrategias de la columna anterior. A continuación describe las competencias que deben desarrollar el estudiante, la estrategia de evaluación y por último los criterios de evaluación.

La apreciación de los docentes frente a la participación en clase como estrategia de evaluación lo realizan de forma oral, lanzando preguntas abiertas al curso y a quien responda la respuesta correcta le colocan puntos que al final del periodo son indicadores para incrementar la calificación final; esta actividad mantiene al estudiante atento y activo durante la clase y genera una calificación complementaria y real frente al aprendizaje.

No obstante, que el proceso de evaluación es necesario dado que en base a esto pueden corregir o cambiar sistemas, métodos o la forma de construir conocimientos, igual que representa un medio por el cual se dan cuenta de quién o quienes están interesados en la asignatura y de éste modo puedan evaluar las metodologías y procesos de enseñanza para que estén unificados los conceptos, así mismo permite realizar el ciclo de "planear, hacer, verificar y ajustar (PHVA) en donde la evaluación es fundamental para verificar que lo que se hace se hace bien y con pertinencia.

Claramente detallan las prácticas evaluativas, ya que les permite realizar diagnósticos de aprendizaje, articulando teoría y práctica, en el que se determina la comprensión y análisis de los contenidos temáticos, las consideran como el desarrollo de avance en la enseñanza a través de 
procedimientos, ejecución y aplicación real que evidencie la efectiva orientación; piensan que las prácticas evaluativas no solamente se remiten al momento del examen, sino que es constante.

Además, explican que los docentes tienen varios criterios de evaluación como lo demuestra a continuación:

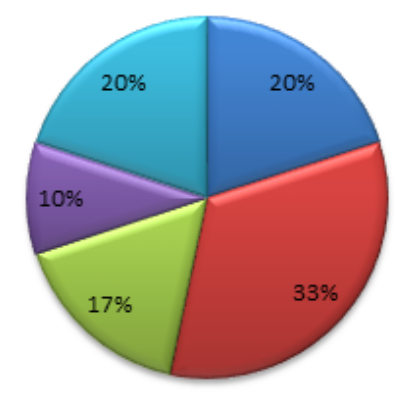

Efectividad

๑ habilidades y agilidad

$\square$ Redacción y acciòn

$\square$ Habilidades y agilidad

$\square$ Conocimientos teóricos

\section{Gráfica 2. Criterios de evaluación.}

Dentro de los criterios que utilizan para realizar las evaluaciones se basan en hechos reales, buscando analizar casos de manera aplicada y así observar si los estudiantes han comprendido los contenidos temáticos de la asignatura, además se basan en la efectividad o confianza en el que toma como referencia el desarrollo del contenido programático graficado en los planes de trabajo académico, justificadas con las propias palabras del estudiante y simultáneamente los criterios de conocimientos teóricos y prácticos.

No obstante, tiene presente la forma de redactar y actuar en referencia a las competencias y componentes del Proyecto Educativo Institucional de la Policía Nacional en cuanto al ser, saber y saber hacer y la habilidad y agilidad de forma individual respectivamente. Es de anotar que un grupo de docentes de la escuela están referenciados para desarrollar el proceso de enseñanza aprendizaje con el Proyecto Educativo Institucional, lo cual demuestra pertinencia institucional y profesionalismo en su labor policial.

Las diferentes concepciones de la evaluación y de las categorías asociadas (calificación, acreditación), así como el sentido y los recursos para llevarla a cabo se han visto impactados por las diversas teorías y enfoques sobre la educación, el currículo, el sujeto como parte del proceso educativo, el aprendizaje y las modalidades de enseñanza. Las nuevas formas de aproximación al 
conocimiento plantean un sentido nuevo en la forma en que se percibe y aplica la evaluación, lo que conlleva a producir espacios de reflexión en torno a las concepciones y prácticas evaluativas que permitan el cambio de paradigmas y fomenten la innovación en la acción pedagógica para crear ambientes educativos que fomenten el desarrollo integral de los estudiantes.

El cambio de paradigmas frente al proceso de concepciones y prácticas evaluativas que predominan en la acción pedagógica de los docentes de la Escuela de Policía Rafael Reyes, se guía mediante las creaciones didácticas evaluativas de la práctica pedagógica, a partir del desarrollo del plan de clase, en el que se favorece la comunidad educativa de la Escuela de Policía Rafael Reyes.

De los análisis realizados acerca de los fundamentos pedagógicos de los enfoques centrados en el aprendizaje se desprende la consideración de que la evaluación es más que un proceso para calcular y asignar calificaciones, como se ha planteado en la perspectiva tradicional de la evaluación limitarse a esta operación implica que solo se están analizando los resultados y no el proceso de aprendizaje, ya que éste considera el lugar de partida, los avances y el punto de llegada del estudiante en su proceso de aprendizaje y así como conocidos los aciertos y desaciertos de los conceptos. Las habilidades evaluativas que los docentes de la escuela utilizan en su ejercicio pedagógico y priorizado los conceptos y prácticas que más utilizan los docentes frente al proceso de evaluación del aprendizaje, se llegó a la necesidad urgente de orientar un curso sobre procesos de evaluación del aprendizaje para los docentes de la unidad y que como resultado final de la capacitación se diseñe una cartilla didáctica, la cual será presentada a las demás escuelas de formación de la Policía Nacional, como guía y fortalecimiento del proceso de evaluación del aprendizaje, cuyos frutos se verán reflejados en la formación policial, en el servicio policial y la seguridad ciudadana. La capacitación es a corto plazo y el diseño de la cartilla a mediano plazo; ya que se pondrá a validación con pares académicos interinstitucionales para su confiabilidad y efectividad.

Con relación a la tercera etapa Rivera (2015) perfilaron la estrategia, la cual surgió de los análisis realizados acerca de los fundamentos pedagógicos de los enfoques centrados en el aprendizaje se desprende la consideración de que la evaluación es más que un proceso para calcular y asignar calificaciones, como se ha planteado en la perspectiva tradicional de la evaluación, limitarse a esta operación implica que solo se están analizando los resultados y no el proceso de aprendizaje, ya que éste considera el lugar de partida, los avances y el punto de llegada del estudiante en su proceso de aprendizaje. 


\section{INTERPRETACIÓN Y ARGUMENTACIÓN DE LOS RESULTADOS}

El modelo pedagógico que maneja la escuela, es el constructivismo, basado en competencias, en donde privilegia el aprendizaje activo, los docentes de la Escuela de Policía Rafael Reyes encuentran dentro del contenido programático de cada asignatura unas estrategias de evaluación, las cuales describen muy bien en el plan de trabajo que se presenta al área académica de la asignatura que va a orientar; aunque al indagar sobre estas se evidenció, que no las aplican en su totalidad.

Los docentes al inicio de la clase explican a los estudiantes el sistema de evaluación que aplicaran dentro del curso, pero no se cumple en su totalidad, puesto que los docentes evalúan a través de trabajos y talleres dentro de la transversalidad de la investigación, algunos docentes utilizan dentro del proceso de evaluación la elaboración de ensayos, sin embargo, se evidenció que existen falencias en el conocimiento sobre la construcción de los mismos.

La opinión de los docentes frente a los resultados del proceso de evaluación de los contenidos programáticos, les permite corregir o cambiar la metodología del proceso de enseñanza, con el fin de unificar conceptos; también les permite conocer el interés del estudiante frente a la asignatura, para los docentes, evaluar representa sacar unos promedios numéricos, en donde se evidencia si el estudiante aprobó la asignatura o no, teniendo en cuenta la planilla de calificaciones que deben entregar en la "secretaria académica" de la Unidad.

La investigación demostró que los docentes en la Unidad evalúan durante todo el período, lo que significa que el proceso es continuo y permanente y los espacios que la escuela brinda para dar a conocer y reflexionar sobre el proceso de evaluación son pocos; sólo se dan en los comités académicos, esto para los docentes de planta.

Los docentes de la Unidad aplican la evaluación formal de tipo cuantitativa, en la que se dedican exclusivamente a obtener las calificaciones requeridas al final de cada periodo.

Sugieren que la capacitación se brinde mediante un seminario taller y como resultado de la capacitación se proyectara una cartilla didáctica, elaborada por los docentes, la cual contenga de forma clara y sencilla estrategias de evaluación del aprendizaje, para que sean aplicadas por toda la comunidad docente de las escuelas de formación de la policía como referente bibliográfico. 
Ellos proponen que la capacitación sea a corto plazo y el diseño de la cartilla a mediano plazo; ya que se pondrá a validación con pares académicos interinstitucionales para su confiabilidad y efectividad.

\section{BIBLIOGRAFÍA}

González, M. (2004). Principios teóricos de evaluación educativa. San José, Costa Rica:

EUNED

Nacional, P. (2010). Doctrina Educativa para el Docente policial. Bogotá, Colombia.

Nacional, P. (2010). Proyecto Educativo Institucional (Vols. 4-1). Bogotá, Colombia.

Rivera, H.v. (2015). Concepciones y Prácticas Evaluativas que Predominan en la Acción

Pedagógica de los Docentes de la Escuela de Policia Rafel Reyes. Resultado de Investigación,

Policia Nacional, Boyacá, Santa Rosa de Viterbo. 